\title{
4. Die Musterauffassung wissenschaftlicher Phänomene
}

Im vorhergehenden Abschnitt wurde herausgearbeitet, dass Phänomene diejenigen Sachverhalte sind, die in der wissenschaftlichen Praxis die Explanandum- und die Belegrolle spielen. In den folgenden beiden Kapiteln soll die Frage diskutiert werden, ob man genauer angeben kann, welche Sachverhalte diese Funktionen übernehmen. Dazu betrachte ich zuerst in Kapitel 4 eine Interpretation der Daten-Phänomen-Unterscheidung, die in der Debatte um Bogens und Woodwards Unterscheidung weit verbreitet ist. Dieser Interpretation zufolge sind Phänomene diejenigen Sachverhalte, die durch Muster in Datensätzen repräsentiert werden. Es wird sich allerdings herausstellen, dass diese Auffassung zurückgewiesen werden muss.

\subsection{Was ist ein Muster?}

Den hier zu diskutierenden Vorschlag bezeichne ich als die Musterauffassung wissenschaftlicher Phänomene. Diese Auffassung wird zum einen durch das oben bereits skizzierte Schmelzpunktbeispiel und zum anderen durch Aussagen wie die folgende aus Woodwards Aufsatz Data and Phenomena motiviert:

"The problem of detecting a phenomenon is the problem of detecting a signal in this sea of noise, of identifying a relatively stable and invariant pattern of some simplicity and generality with recurrent features - a pattern which is not just an artifact of the particular detection techniques we employ or the local environment in which we operate." ${ }^{\prime 1}$

Autoren wie James McAllister, Bruce Glymour oder Mieke Boon gehen davon aus, dass die gerade zitierte Aussage besagt, dass Phänomene von Mustern in Datensätzen repräsentiert werden. ${ }^{32}$ Der einfachste Fall, an dem man sich klarmachen kann, was mit dem Ausdruck ,Muster in Datensätzen“ gemeint ist, besteht darin, graphische Darstellungen von Daten zu betrachten. Man denke an ein Koordinatensystem, in dem die Datenpunkte

\footnotetext{
${ }^{31}$ Woodward (1989), S. 396-397, meine Hervorhebung.

${ }^{32}$ Vgl. McAllister (1997), Glymour (2000), Boon (2008).
} 
aufgetragen werden. Ein Muster ist in einer solchen Darstellung eine glatte Kurve $^{33}$, die man durch die Datenpunkte legt. Muster sind dieser Auffassung zufolge das, was man auch als Datenmodelle bezeichnet:

„A model of data is a corrected, rectified, regimented, and in many instances idealized version of the data we gain from immediate observation, the so-called raw data. Characteristically, one first eliminates errors (e.g. removes points from the record that are due to faulty observation) and then presents the data in a ,neat' way, for instance by drawing a smooth curve through a set of points." ${ }^{34}$

Die Rede davon, dass Muster in Datensätzen Phänomene repräsentieren, besagt somit zum einen, dass die Muster (graphische) Repräsentationen der Ausprägung bestimmter Eigenschaften sind, und zum anderen, dass man davon ausgeht, das entsprechende Muster nicht nur in der betrachteten Stichprobe, sondern in der gesamten Population zu finden. Gemäß der Musterauffassung sind Phänomene demnach entweder das, was der Statistiker als Populationskennwerte bezeichnet, oder funktionale Zusammenhänge, die das Verhalten einer Population beschreiben. ${ }^{35}$ Für die Bestimmung solcher Populationskennwerte bzw. der jeweiligen funktionalen $\mathrm{Zu}-$ sammenhänge gibt es wohletablierte mathematisch-statistische Verfahren. ${ }^{36}$ Entsprechend führt die Musterauffassung ein epistemisches Kriterium dafür an, dass ein Sachverhalt unter den Phänomenbegriff fällt, nämlich, dass Wissen über das Phänomen durch mathematisch-statistische Interpretation beobachteter Daten gewonnen werden kann. ${ }^{37}$ Legt man diese

${ }^{33}$ Eine Kurve bezeichnet man als glatt, wenn sie durch eine stetige und stetig differenzierbare Funktion beschrieben werden kann.

${ }^{34}$ Frigg und Hartmann (2006). Der Terminus wurde von Patrick Suppes (1962) in die wissenschaftstheoretische Diskussion eingeführt.

${ }^{35}$ Ein Beispiel für ersteres ist der Schmelzpunkt von Blei, ein Beispiel für letzteres ist die periodische Änderung der Radialgeschwindigkeit eines Sterns, wie sie in Abschnitt 4.2 thematisiert wird.

${ }^{36}$ Diese Verfahren selbst sind natürlich auch aus philosophischer Perspektive interessant, wie man beispielsweise am sog. Kurvenanpassungsproblem sieht, das in Kapitel 11 thematisiert wird.

${ }^{37}$ Natürlich stellt sich an dieser Stelle auch die Frage, ob Bogen und Woodward selbst der Musterauffassung wissenschaftlicher Phänomene zustimmen würden. Die oben genannten Autoren gehen hiervon aus. Meiner Meinung nach ist dies allerdings nicht korrekt. Dies kann man beispielsweise daran erkennen, dass Bogen und Woodward auch die Existenz schwacher neutraler Ströme als Phänomen ansehen. Gemäß der Musterauffassung wäre dieser Sachverhalt aber kein Phänomen, da er mit keinem Muster in einem Datensatz korrespondiert. Aber solche exegetischen Fragen sind für 
Auffassung wissenschaftlicher Phänomene zugrunde ergibt sich folgendes Wissenschaftsbild:

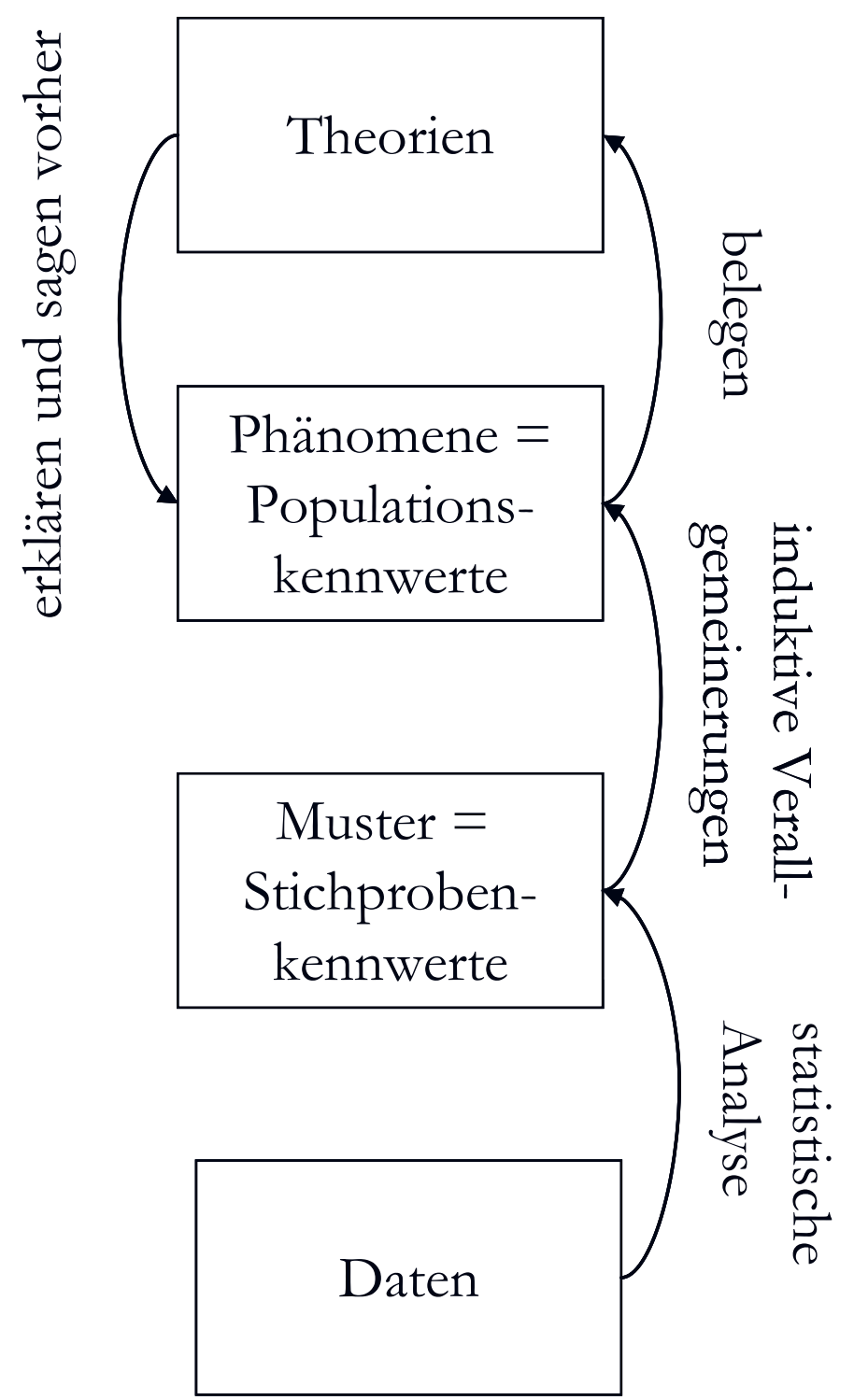

Abbildung 3: Die Musterauffassung wissenschaftlicher Phänomene

An dieser Stelle lohnt es sich, auf einen Einwand hinzuweisen, den Bruce Glymour gegen die Daten-Phänomen-Unterscheidung vorgebracht hat. Glymours Einwand geht von der Musterauffassung wissenschaftlicher Phänomene aus und lautet wie folgt: Wenn Phänomene nichts anderes als Populationskennwerte sind, dann tun Bogen und Woodward letztlich nichts

meine Untersuchung von untergeordneter Wichtigkeit. Für mich rechtfertigt bereits die Tatsache, dass es einige Autoren gibt, die die Musterinterpretation vorschlagen, eine kritische Prüfung derselben. 
anderes als einer wohlbekannten Unterscheidung aus der Statistik einen neuen Namen zu geben. Anstelle von Stichproben- und Populationskennwerten sprechen sie von Daten und Phänomenen. Aber hierbei handele es sich bloß um eine unnötige und im schlimmsten Fall Verwirrung stiftende terminologische Reform, die keinerlei neue Einsichten ermögliche. Aus diesem Grund sei die Daten-Phänomen-Unterscheidung philosophisch uninteressant. ${ }^{38}$ Wäre Glymours Argument schlüssig, würde dies von vornherein auch das in meiner Arbeit verfolgte Projekt ad absurdum führen. Glücklicherweise wird sich aber im kommenden Abschnitt herausstellen, dass Glymours Einwand nicht stichhaltig ist. Dort möchte ich anhand eines Beispiels aufzeigen, dass die Musterauffassung wissenschaftlicher Phänomene nicht geeignet ist, um den Phänomenbegriff zu explizieren. Dies führt wiederum dazu, dass Glymours Kritik an Bogens und Woodwards Unterscheidung zurückgewiesen werden kann, denn diese setzt die Musterauffassung voraus. Nichtsdestotrotz bleibt die grundsätzliche Frage, die Glymour aufwirft, beachtenswert: Inwiefern ist die Daten-PhänomenUnterscheidung überhaupt philosophisch interessant? Diese Frage werde ich im Teil B dieser Arbeit aufgreifen und dort versuchen, sie im Hinblick auf erkenntnistheoretische Fragen, die sich im Rahmen der Realismusdebatte stellen, zu beantworten.

\subsection{Ein Einwand gegen die Musterauffassung: der Fall extrasolarer Planeten}

In diesem Abschnitt möchte ich anhand eines Beispiels aus der Astronomie aufzeigen, dass die Musterinterpretation von Bogens und Woodwards Unterscheidung keine geeignete Explikation des Phänomenbegriffs liefert. ${ }^{39}$ Mein Fallbeispiel ist die erste experimentelle Bestätigung der Existenz eines extrasolaren Planeten im Jahr 1995. Die Existenz extrasolarer Planeten sollte, so meine These, als Phänomen im Sinne der Daten-PhänomenUnterscheidung aufgefasst werden, obwohl dieser Sachverhalt nicht im oben beschriebenen Sinne mit einem Muster in einem Datensatz korrespondiert.

\footnotetext{
${ }^{38}$ Vgl. Glymour (2000), S. 33-34.

${ }^{39}$ Obgleich es der Fall ist, dass einige Phänomene tatsächlich mit Mustern in Datensätzen korrespondieren. Hierbei handelt es sich allerdings um eine Unterklasse der Phänomene.
} 
Als „extrasolare Planeten“ oder kurz „Exoplaneten“ bezeichnet man Planeten außerhalb unseres Sonnensystems. In der Astronomie war es lange Zeit eine offene Forschungsfrage, ob auch andere Sterne neben unserer Sonne über Planetensysteme verfügen. Diese Frage faszinierte Astronomen und Philosophen seit jeher, nicht zuletzt deswegen, weil sie eng mit der Frage nach der Möglichkeit von Leben nicht-irdischen Ursprungs im Universum verknüpft ist. Ein weiterer wichtiger Grund für das Interesse der Astronomen an Exoplaneten war und ist, dass die Entdeckung von extrasolaren Planeten einen wichtigen Beleg für bestimmte Theorien zur Entstehung von Planeten und Sternen darstellt, die die Existenz solcher Planeten vorhersagen. ${ }^{40}$ Um den letzten Punkt zu verdeutlichen, skizziere ich kurz die Grundideen einiger Theorien zur Planetenentstehung, die in den letzten 150 Jahren in der Astronomie vertreten wurden. Ich werde es allerdings bei einer sehr groben Skizze belassen und gehe nicht darauf ein, wie diese Theorien im Detail aussehen und welche Gründe zur Ablösung einer Theorie durch die andere geführt haben.

$\mathrm{Ob}$ man die Existenz von extrasolaren Planeten für wahrscheinlich hält, hängt eng damit zusammen, ob man die Struktur unseres Sonnensystems, in dem es verschiedene Planeten gibt, als Produkt einer durchschnittlichen Sternenentwicklung oder als einen seltenen kosmischen Zufall ansieht. Dies wiederum hängt davon ab, welche Erklärung man für die Entstehung von Planeten akzeptiert. Bis zum Ende des 19. Jahrhunderts wurde in der Astronomie weithin die sog. Nebulartheorie der Planetenentstehung akzeptiert, die maßgeblich von Laplace entwickelt wurde. Planeten entstehen gemäß dieser Theorie in einem heißen Nebel, der die Sterne bei ihrer Entstehung umgibt. Die Entstehung von Planetensystemen ist der Nebulartheorie zufolge eine direkte Folge der Sternentstehung und aus diesem Grunde müsste es gemäß dieser Theorie viele Planeten bei anderen Sternen geben. Gegen Ende des 19. Jahrhunderts wurde die Nebulartheorie jedoch durch eine andere Auffassung abgelöst, die davon ausging, dass unser Sonnensystem das Produkt einer seltenen kosmischen Katastrophe ist. Die Entstehung von Planeten erklärte man sich nun so: Zwei Sterne seien sich auf ihrem Weg durchs All zu nahe gekommen und hätten sich durch die starke Gravitationswechselwirkung gegenseitig Materie ,,ausgerissen“. Aus diesen Materieklumpen seien die Planeten entstanden. Aufgrund der großen Entfernungen zwischen den Sternen im Universum sei es statistisch äußerst unwahrscheinlich, dass sich Sterne begegnen und so nahe kommen,

${ }^{40}$ Vgl. z.B. Casoli und Encrenaz (2007), S. 91-116. 
wie es bei diesem kosmischen Ereignis der Fall war. Aber diese Theorie konnte sich nicht durchsetzen. Nach 1940 wurde die Grundidee der Theorie von Laplace wieder aufgegriffen und weiter ausgearbeitet. Planeten entstehen dieser „modifizierten Nebulartheorie“ zufolge in Gas- und Staubscheiben, die ein gewöhnliches Beiprodukt der Sternentstehung sind. Planetenentstehung ist im Rahmen dieser Theorie daher wieder Teil einer typischen Sternentwicklung. Die aktuell in der Astronomie vertretenen Theorien gehören ebenfalls zu diesem Theorietypus.

Vor diesem Hintergrund wird klar, warum Astronomen immer wieder versuchten, extrasolare Planeten aufzuspüren. Eine solche Entdeckung wäre ein wichtiger Beleg für bzw. gegen unterschiedliche Theorien der Planeten- und Sternentstehung. Jedoch waren die Bemühungen der Astronomen lange Zeit nicht von Erfolg gekrönt, denn bei der Suche nach den Exoplaneten sieht man sich mit erheblichen Schwierigkeiten konfrontiert: Exoplaneten sind weit von der Erde entfernt, relativ klein und leuchtschwach (sie reflektieren nur einfallendes Sternenlicht, leuchten aber nicht selbst). Es ist deshalb nicht möglich, sie mit bloßem Auge oder durch ein Teleskop zu beobachten. Aus diesem Grund begann man mittels verschiedener sog. indirekter Methoden nach Exoplaneten zu suchen. Diese Methoden zielen darauf ab, die Existenz von Exoplaneten zu erschließen, indem man ihren kausalen Einfluss auf andere Himmelskörper nachweist. ${ }^{41}$

Bereits 1897 verkündete der Astronom Thomas See gleich mehrere Exoplaneten gefunden zu haben, da er Verdunklungen auf der Oberfläche der Fixsterne beobachtet habe, die er auf Planeten zurückführte, die die Fixsterne passierten. Diese vermeintlichen Planetenentdeckungen konnten jedoch nicht bestätigt werden. Sees angebliche Planetenfunde waren jedoch nur der erste Schritt einer ganzen Reihe von vermeintlichen Nachweisen von Exoplaneten, die allesamt keiner genaueren Prüfung standhielten. Berühmt (zumindest innerhalb der Astronomie) wurde beispielsweise der vermeintliche Nachweis eines Planeten bei Barnards Pfeilstern durch den niederländischen Astronomen Piet van de Kamp, der auf systematische Fehler des verwendeten Teleskops zurückführbar ist. ${ }^{42}$ Die regelmäßige Meldung von Planetenfunden, die sich nicht bestätigen ließen, führte dazu, dass noch 1995, kurz vor der Bekanntgabe des ersten bestätigten Nachwei-

${ }^{41}$ Eine solche Methode, die sog. Radialgeschwindigkeitsmethode, stelle ich im weiteren Verlauf dieses Abschnitts kurz vor. Einen Überblick über entsprechende Messverfahren liefern z.B. Casoli und Encrenaz (2007), Kapitel 2 oder Kosso (2006).

${ }^{42}$ Vgl. z.B. Casoli und Encrenaz (2007), S. 18-20. 
ses eines Exoplaneten durch Michael Mayor und Didier Queloz, der Astronom David C. Black schrieb:

„However, the results to date are that no other planetary system has been detected, and the absence of detection is becoming statistically significant, particularly as it relates to the existence of brown dwarf companions to mainsequence stars." 43

Die Waage drohte demnach wieder in Richtung derjenigen Theorien zu kippen, die Planeten als Produkte seltener kosmischer Konstellation betrachten. Schon kurz nach der Veröffentlichung von Blacks Artikel, am 06. Oktober 1995, änderte sich die Situation jedoch grundlegend. An diesem Tag gaben Mayor und Queloz beim Cambridge Workshop on Cool Stars, Stellar Systems and the Sun in Florenz, dem sog. „Cool Star Meeting“, die Entdeckung eines Planeten, der um den Stern 51 Pegasi kreist, bekannt. ${ }^{44}$ Ihre Entdeckung konnte bald von anderen Forscherteams bestätigt werden und wurde rasch in der wissenschaftlichen Gemeinschaft anerkannt. Den entdeckten Planet bezeichnet man als „51 Pegasi b“ oder auch als „Bellerophon“.

Wenn man nun die Frage stellt, wie die Unterscheidung zwischen Daten und Phänomenen auf die erste Entdeckung eines Exoplaneten angewendet werden kann, muss man einen Blick auf die Detektionsmethode werfen, mit der Mayor und Queloz diesen Planeten entdeckt haben. Diese Methode beruht auf der Messung der Radialgeschwindigkeit des Sterns 51 Pegasi. Als Radialgeschwindigkeit bezeichnet man die Komponente der Geschwindigkeit eines sich bewegenden Objekts, deren Vektor genau parallel zur Sichtlinie des Beobachters liegt, d.h. der genau auf einen Beobachter zu- bzw. von ihm wegzeigt. Mayor und Queloz führten Messungen der Radialgeschwindigkeit von 51 Pegasi durch. Genauer gesagt misst man bei der Radialgeschwindigkeitsmethode nicht direkt die Änderung der Radialgeschwindigkeit, sondern vielmehr die Dopplerverschiebung des Lichts, das der Stern emittiert. Aus dieser lässt sich dann die Radialgeschwindigkeit errechnen. Diesen Zwischenschritt beachte ich allerdings im Folgenden aus Gründen der Einfachheit nicht, da er für meine Argumentation keinen Unterschied macht. ${ }^{45}$

\footnotetext{
${ }^{43}$ Black (1995), S. 359, Hervorhebungen im Original.

${ }^{44}$ Vgl. auch Mayor und Queloz (1995).

45 Eine genauere Beschreibung der Methode findet man beispielsweise in Casoli und Encrenaz (2007), S. 22-30. Vorgeschlagen wurde diese Methode bereits in den 1950er
} 
Mayor und Queloz stellten im Verlauf ihrer Messungen fest, dass sich die Radialgeschwindigkeit von 51 Pegasi periodisch mit der Zeit ändert. Ausgehend von dieser periodischen Änderung erschlossen die Astronomen die Existenz eines Exoplaneten, der 51 Pegasi umkreist und durch Gravitationswechselwirkung mit dem Stern die Änderung der Radialgeschwindigkeit verursacht. Betrachtet man die folgende Abbildung, so scheint man es hier mit einem paradigmatischen Fall für die Musterauffassung wissenschaftlicher Phänomene zu tun zu haben.

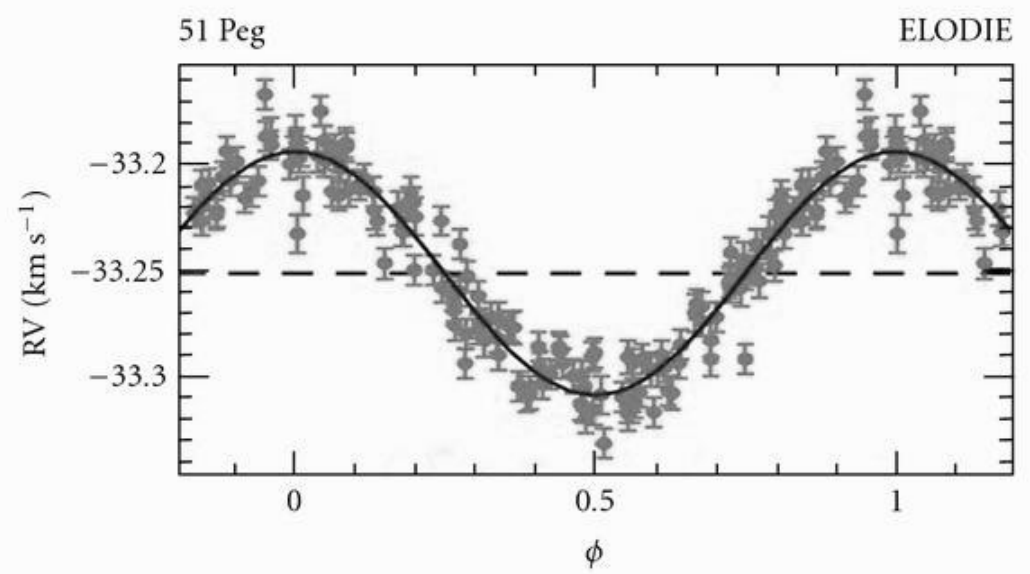

Abbildung 4: Radialgeschwindigkeitsänderung von 51 Pegasi (aus: Cuesta et al. 2010, S. 2)

Die Abbildung zeigt eine Verteilung von Datenpunkten, durch die eine Sinuskurve gelegt wurde. Mayor und Queloz haben bei ihren Messungen eine Vielzahl an Daten gesammelt. Auf Grundlage mathematischstatistischer Techniken führten sie eine sog. Kurvenanpassung durch und identifizierten auf diese Weise in ihren Daten ein regelmäßiges Muster. Man hat es bei diesem Muster nicht mehr, wie z.B. beim Schmelzpunkt von Blei, mit einer eindimensionalen Häufigkeitsverteilung zu tun, sondern mit einem funktionalen Zusammenhang zwischen zwei Merkmalen: der Radialgeschwindigkeit (auf der Ordinatenachse) und einer auf die Umlaufzeit des Planeten normierten Zeitangabe (auf der Abszissenachse). Der genaue funktionale Zusammenhang wird mit Hilfe der Regressionsrechnung bestimmt. Am Ende dieses Kurvenanpassungsprozesses steht das in der

Jahren von dem deutsch-baltischen Astronomen Otto Struve. Vgl. Struve (1952). Es dauerte allerdings bis in die 1990er Jahre, bis die verfügbaren Teleskope und Detektoren leistungsfähig genug waren, um für einen entsprechenden Nachweis geeignet zu sein. 
Graphik eingezeichnete Muster. Dieses Muster repräsentiert die regelmäßige Änderung der Radialgeschwindigkeit des Sterns. Gemäß der Musterauffassung müsste man nun sagen, dass das einzige Phänomen, das Mayor und Queloz entdeckt haben, die periodische Änderung der Radialgeschwindigkeit von 51 Pegasi ist, da dieser Sachverhalt der einzige ist, der in diesem Kontext mit einem Muster in einem Datensatz korrespondiert. ${ }^{46}$ Gegen diese Auffassung möchte ich im Folgenden argumentieren. Es soll plausibel gemacht werden, dass es letztlich ein anderes Phänomen war, das Mayor und Queloz entdeckt und für das sie sich vornehmlich interessiert haben, nämlich die Existenz extrasolarer Planeten. Damit möchte ich nicht behaupten, dass die periodische Änderung der Radialgeschwindigkeit von 51 Pegasi kein wissenschaftliches Phänomen ist. Ich möchte nur dafür argumentieren, dass die Musterauffassung wissenschaftlicher Phänomene nicht allgemein genug ist, um zur Explikation des Phänomenbegriffs geeignet zu sein. Welche Gründe sprechen nun dafür, dass die Existenz extrasolarer Planeten als Phänomen angesehen werden sollte?

Erstens ist es der Sachverhalt, dass Exoplaneten existieren, der die funktionalen Rollen inne hat, die im Abschnitt 4 als charakteristisch für Phänomene identifiziert wurden: Dass es extrasolare Planeten gibt, liefert einen wichtigen Beleg für astronomische Theorien über Stern- und Planetenentstehung und gleichzeitig ist es dieser Sachverhalt, der durch solche Theorien erklärt werden kann, sobald diese Theorien hinreichend gut etabliert sind. Die Änderung der Radialgeschwindigkeit von 51 Pegasi allein liefert hingegen noch keinen Beleg für die gerade erwähnten Theorien, denn diese Änderung hätte auch durch andere Ursachen als den gravitativen Einfluss eines Planeten hervorgerufen werden können. Zum Beispiel hätte das entsprechende Muster auch durch ein Pulsieren des Sterns oder sog. SpotRotation hervorgerufen werden können. ${ }^{47}$ Diese alternativen Ursachen mussten von den Astronomen in Betracht gezogen und durch weitere Überlegungen ausgeschlossen werden. Wissenschaftler sind an Belegen interessiert, die gute Gründe für oder gegen die Akzeptanz bestimmter Hypothe-

\footnotetext{
${ }^{46}$ Streng genommen müsste man in diesem Fall sogar sagen, dass das Phänomen darin besteht, dass die Spektrallinien des von 51 Pegasi emittierten Lichts in regelmäßiger Weise alternierend in Richtung des roten und des blauen Endes des Spektrums verschoben werden.

${ }^{47}$ Hierbei handelt es sich nicht um bloß logische Möglichkeiten, vorgebracht von einem Philosophen, der für irgendeine Form von empirischer Unterbestimmtheit argumentieren will, sondern um relevante Möglichkeiten, die von Astronomen ernsthaft in Betracht gezogen wurden. Vgl. Mayor und Queloz (1995), S. 357-358.
} 
sen an die Hand geben und die Radialgeschwindigkeitsänderung alleine liefert noch keinen solchen guten Grund. Über einen guten Grund verfügt man vielmehr erst, wenn die relevanten Alternativhypothesen ausgeschlossen werden können. Aber damit tut man nichts anderes als die Exoplanetenhypothese zu etablieren. ${ }^{48}$

Zweitens passt es zur alltagssprachlichen und zur wissenschaftlichen Verwendungsweise des Ausdrucks „Phänomen“, die Existenz von Exoplaneten als solches zu bezeichnen. Im alltäglichen Sprachgebrauch sind Phänomene Sachverhalte, die überraschend, beachtenswert, manchmal außergewöhnlich sowie erklärungsbedürftig sind und die deshalb unser Interesse hervorrufen - und im hier verhandelten Beispiel war es auch die Existenz extrasolarer Planeten, für die sich die Astronomen letztlich interessierten und nicht das Radialgeschwindigkeitsmuster. Zudem beziehen sich auch Astronomen mit dem Ausdruck „Phänomen“ auf extrasolare Planeten bzw. ihre Existenz, wie folgendes Zitat aus einem Standardlehrbuch für Astrophysik beispielhaft zeigt:

„A proper understanding of phenomena like black holes, quasars and extrasolar planets requires that we understand all physics that underlies all of astrophysics." 49

Drittens spielte es für die Astronomen keine entscheidende Rolle, welche der verschiedenen vorhandenen Methoden zum Nachweis von Exoplaneten tatsächlich benutzt wurde, um einen solchen Planeten aufzuspüren. Andere Nachweismethoden, wie z.B. photometrische oder astrometrische, wären dazu ebenso geeignet gewesen. Aber diese Methoden hätten völlig andere Arten von Daten hervorgebracht und folglich auch völlig andere Muster in Datensätzen.

Wie ich oben bereits erwähnt habe, möchte ich mit diesen Ausführungen nicht bestreiten, dass die Radialgeschwindigkeitsänderung von 51 Pegasi ein Phänomen ist, ich argumentiere lediglich dafür, dass sie im hier betrachteten Kontext nicht das einzige Phänomen ist. Das relevante Phäno-

${ }^{48}$ Diese Überlegung weist auf bestimmte Unzulänglichkeiten sog. inkrementeller Belegkonzeptionen hin, denen zufolge die Belegrelation in positiver wahrscheinlichkeitstheoretischer Relevanz zwischen Beleg und zu Belegendem besteht. Eine philosophische Argumentation dafür, dass solche Konzeptionen tatsächlich nicht hinreichend sind, um den Belegbegriff zu explizieren, liefert Peter Achinstein (2001), S. 5-10. Ich werde auf diesen Punkt außerdem in Abschnitt 5.2.2 zurückkommen.

${ }^{49}$ Duric (2003), S. i. 
men in diesem Kontext ist vielmehr die Existenz extrasolarer Planeten. Dies bedeutet wiederum, dass wir, um ein Phänomen aufzuspüren, häufig Schlüsse von Mustern in Datensätzen, wie der periodischen Radialgeschwindigkeitsänderung eines Sterns, auf die Ursachen für solche Muster, wie die Existenz eines Exoplaneten, vollziehen müssen.

Zusammenfassend lassen sich aus der Diskussion der Musterauffassung zwei Schlussfolgerungen ziehen: Erstens wird klar, dass die Musterauffassung keine adäquate Explikation des Phänomenbegriffs ermöglicht. Nicht alle Phänomene korrespondieren mit Mustern in Datensätzen. Allerdings ist es so, dass die Phänomene, die durch Muster in Datensätzen repräsentiert werden, eine Teilklasse der wissenschaftlichen Phänomene ausmachen. Da diese Teilklasse im Teil C dieser Arbeit an verschiedenen Stellen eine wichtige Rolle spielen wird, möchte ich für sie eine eigene Benennung einführen: Ich werde sie im Folgenden als Musterphänomene bezeichnen. Musterphänomene sind solche Phänomene, bei denen beobachtbaren $\mathrm{Ob}$ jekten (wie z.B. Blei) eine beobachtbare Eigenschaft (wie z.B. das Haben einer bestimmten Schmelztemperatur) zugeschrieben wird, die genaue Ausprägung dieser Eigenschaft aber mittels mathematisch-statistischer Methoden erschlossen werden muss, indem ein Muster in einem Datensatz identifiziert wird. Abgegrenzt werden Musterphänomene von direkt beobachtbaren Phänomenen (wie z.B. dem Phänomen, dass der Himmel blau ist) und Phänomenen, die als Ursachen für Musterphänomene erschlossen werden (wie dem Phänomen extrasolarer Planeten). ${ }^{50}$ Zweitens resultiert aus diesen Überlegungen, dass Glymours Argumentation dafür, dass die Daten-Phänomen-Unterscheidung überflüssig und philosophisch uninteressant ist, nicht zu überzeugen vermag. Schließlich beruht diese Argumentation wesentlich auf der Musterauffassung wissenschaftlicher Phänomene. ${ }^{51}$

\footnotetext{
${ }^{50}$ Uljana Feest trifft die gleiche Unterscheidung. Sie bezeichnet Musterphänomene als Oberflächenphänomene (,surface phenomena“) und Phänomene, die als Ursachen für Muster erschlossen werden, als versteckte Phänomene (,hidden phenomena“). Vgl. Feest (2009). Den Terminus ,verstecktes Phänomen“ werde ich im Folgenden übernehmen.

51 An dieser Stelle soll noch einmal darauf hingewiesen werden, dass diese Zurückweisung von Glymours Kritik auf der Voraussetzung beruht, dass Bogens und Woodwards Unterscheidung tatsächlich eine deskriptive Unterscheidung ist, die die Bedeutung des Phänomenbegriffs erhellen soll. Denn nur unter dieser Voraussetzung ist die Musterauffassung und damit auch Glymours Argument defizitär. Würden Bogen und Woodward jedoch einen stipulativen Ansatz verfolgen, könnte Glymour hingegen gerechtfertigterweise den philosophischen Wert ihrer Unterscheidung in Zweifel ziehen.
} 
